\title{
Laparoscopic Circumcaval Ureteral Repair with Concomitant Laparoscopic- Guided Nephroscopic Calculi Extraction
}

\author{
L. Curcio, B. Cançado, A. C. Cunha, J. Renteria, F. Gusmão, G. Di Biase \\ Section of Urology, Ipanema General Hospital, Rio de Janeiro, RJ, Brazil
}

\begin{abstract}
Introduction: The pre-ureteral vena cava anomaly, erroneously termed the retrocaval or circumcaval ureter, is a relative rare condition of extrinsic ureteral obstruction. Despite being more common on the right kidney, when present on the left side, it is usually associated with sites inverses. The laparoscopic approach, with all of its know advantages, has good results when used to treat this congenital anomaly. The present surgical video shows a ureteral transposition with ureteropyolostomy, associated with a nephroscopic calyceal calculi extraction. Case Report: A 54 years old man, with discomfort in the right flank and abdominal echography showing a pelvicalyceal dilatation of this side and a calculus of $1.5 \mathrm{~cm}$ in a lower calyx. An intravenous pyelogram and abdominal computed tomography showed an S-shaped (Atkinson classification) pre-ureteral vena cava, and a mid-ureteral extrinsic stricture associated with it. There were no patient pre-operative comorbities and a percutaneous nephrolithotripsy was done 4 years prior on the right kidney. In the present video, a transperitoneal laparoscopic approach was conducted, with 4 trocars. In a stepwise fashion, the ureter sub- and supracaval was gently dissected, sectioned near the renal pelvis, transposed, and spatulated .A ureteropyelostomy was done over a double J stent, which was placed in an antegrade manner. After the posterior wall anastomosis completed, we performed a nephroscopy through the left hand trocar and the calyceal stone was removed by percutaneous stone instrumentation.

Result: The surgery lasted 240 minutes, with minimum blood loss. The diet was resumed on the first postoperative day and he was discharged home on the third postoperative day. After 8 months of follow up, the patient remains asymptomatic and his excretory urography shows good drainage of the right kidney as well a diuretic renography reveals good response following 20 minutes after furosemide intravenous administration. Furthemore, the pelvicalyceal dilatation improved and there are no subsequent calculi.

Conclusion: The laparoscopic approach to circumcaval ureteral anomalies is a feasible option, with concomitant endourologic procedures possible with good results.
\end{abstract}

Int Braz, J Urol. 2009; 35 (Video \#2): 753_4

Available at:www.brazjurol.com.br/videos/november_december_2009/Curcio_753_754video.htm

Accepted:

November 8, 2009

\section{Correspondence address:}

Dr. Lessandro Curcio Gonçalves

Section of Urology

Ipanema General Hospital

Rio de Janeiro, RJ, Brazil

E-mail: lessandrocg@ig.com.br 


\section{EDITORIAL COMMENT}

In this surgical video by Curcio et al., a laparoscopic approach to a circumcaval ureter repair is performed with concomitant stone extraction using endoscopic instrumentation placed through a laparoscopic port. I must commend the authors for this very practical approach to this infrequent clinical presentation. Minimally invasive surgery plays an increasing role in urology, with this video nicely demonstrating how this can be applied to challenging cases such as this in which intracorporeal suturing is required. With the increasing role of robotics in our specialty, I suspect patients presenting with similar clinical presentations in the future can be approached using a robotic assisted laparoscopic approach although whether this will truly improve patient outcome as compared to a pure laparoscopic approach can be debated. The likely merits of a ro- botic assisted approach will be to facilitate intracorporeal suturing, provide 3-dimensional vision for the surgeon while improving ergonomics and decreasing surgeon fatigue.

The important message for our readership is that our surgical specialty is evolving at such an exponential rate that minimally invasive surgical approaches as is demonstrated nicely in this surgical video have a clear and established role. Furthermore, this video integrates multiple surgical instrumentation and approaches to address several concomitant clinical issues (i.e. an obstructed circumcaval ureter and nephrolithiasis) in an attempt to improve treatment related outcome and minimize perioperative morbidity. The impedus will lie on the urologic community to determine the optimal way on how these evolving technologies can be integrated.

Dr. Philippe E. Spiess H Lee Moffitt Cancer Center

Video Section Editor

International Braz J Urol

E-mail:Philippe.Spiess@moffitt.org 